This is a postprint version of the following published document:

Sánchez-Arriaga, G., et al. Kinetic features and non-stationary electron trapping in paraxial magnetic nozzles, in: Plasma sources science and technology, 27(3, 035002), pp. 1-13 (March 2018) DOI: https://doi.org/10.1088/1361-6595/aaad7f

(C) 2018 IOP Publishing Ltd. 


\title{
Kinetic features and non-stationary electron trapping in paraxial magnetic nozzles
}

\author{
G. Sánchez-Arriaga ${ }^{1}$, J. Zhou ${ }^{1}$, E. Ahedo ${ }^{1}$, M. \\ Martínez-Sánchez ${ }^{2}$, J.J. Ramos ${ }^{1,3}$ \\ ${ }^{1}$ Equipo de Propulsion Espacial y Plasmas (EP2), Universidad Carlos III de Madrid, \\ 28911 Leganés, Spain \\ ${ }^{2}$ Department of Aeronautics and Astronautics, Massachusetts Institute of \\ Technology, Cambridge, 02139, Massachusetts, USA \\ ${ }^{3}$ Plasma Science and Fusion Center, Massachusetts Institute of Technology, \\ Cambridge, 02139, Massachusetts, USA \\ October 2017
}

\begin{abstract}
The paraxial expansion of a collisionless plasma jet into vacuum, guided by a magnetic nozzle, is studied with an Eulerian and non-stationary Vlasov-Poisson solver. Parametric analyses varying the magnetic field expansion rate, the size of the simulation box, and the electrostatic potential fall are presented. After choosing the potential fall leading to a zero net current beam, the steady states of the simulations exhibit a quasi-neutral region followed by a downstream sheath. The latter, an unavoidable consequence of the finite size of the computational domain, does not affect the quasi-neutral region if the box size is chosen appropriately. The steady state presents a strong decay of the perpendicular temperature of the electrons, whose profile versus the inverse of the magnetic field does not depend on the expansion rate within the quasi-neutral region. As a consequence, the electron distribution function is highly anisotropic downstream. The simulations revealed that the ions reach a higher velocity during the transient than in the steady state and their distribution functions are not far from mono-energetic. The density percentage of the population of electrons trapped during the transient, which is computed self-consistently by the code, is up to $25 \%$ of the total electron density in the quasi-neutral region. It is demonstrated that the exact amount depends on the history of the system and the steady state is not unique. Nevertheless, the amount of trapped electrons is smaller than the one assumed heuristically by kinetic stationary theories.
\end{abstract}

PACS numbers: 52.75.Di,52.25.Xz, 52.65.Ff

Keywords: electric propulsion, magnetic nozzles, electron trapping Submitted to: Plasma

Sources Sci. Technol. 


\section{Introduction}

Plasma expansions in the presence of magnetic fields appear in astrophysical scenarios, such us pulsars [1], stellar winds [2] and supernova remmants [3], and in laser-produced laboratory plasmas $[4,5]$. They have also engineering applications in electric propulsion. One of the most relevant is a magnetic nozzle [6], where a magnetic field generated by coils is used to guide, expand, and accelerate the plasma without using any physical wall. Several types of thrusters under development, including the helicon plasma thruster [7], the magnetoplasmadynamic thruster [8], and the Variable Specific Impulse Magnetoplasma Rocket (VASIMR) [9] involve magnetic nozzles. Plasma flows in magnetic nozzles have been characterized in the laboratory by using laser-induced fluorescence techniques [10], Langmuir and Mach probes [11] and spectroscopic methods [12] among others.

The modeling of magnetic nozzles has also attracted great attention. One [13], two $[14,15,16,17]$ and three [18] dimensional fluid models have been developed, and the transformation of the internal energy of the plasma into directed kinetic energy, the plasma detachment, and the role played by the plasma-induced magnetic field have been discussed. However, since plasma flows are generally weakly collisional, simple closures of the fluid equation hierarchy for the pressure tensor and the heat fluxes are doubtful. A self-consistent determination of these magnitudes needs inevitably a kinetic description of the plasma.

Stationary solutions of the Vlasov equation in a magnetized plasma expansion have been obtained recently [19]. After assuming steady conditions, a slender nozzle geometry, and a fully magnetized plasma, the conservation of the total energy and the magnetic moment were used to write rigorously the densities of the particles connecting with the source as functions of the electrostatic potential and to compute the latter. It was then found that there exist regions in phase space not connected with either the source or the downstream region where doubly-trapped bouncing particles can exist. Since in collisionsless plasmas the filling of that regions happens during the transient, a stationary model cannot characterize rigorously the (doubly) trapped particles. The plasma spatial solution and its numerical convergence turned out to be very sensitive to the distribution of trapped particles on the divergent side of the nozzle. After adding an heuristic population of trapped electrons, the authors found numerical solutions with the electron density dominated by the confined electrons over most of the divergent jet.

Discussions on whether or not trapped populations of electrons are an essential component of the solutions and how they are determined also arise in other areas of

plasma physics. For instance, for an electron-attracting Langmuir probe in flowing plasma, it was argued that a population of electrons should exist at the ram side of the probe [20]. The formation of such a trapped population during the transient phase, which is an adiabatic process, has been observed recently in non-stationary direct (eulerian) Vlasov simulations [21]. Adiabatic trapping in slowly varying timedependent electric fields [22] has been considered in analytical studies of magnetized 
plasma expansion [23]. Particles can also be trapped due to collisional effects.

Particle collisions, which can also produce trapped particles, have been included in non-stationary particle-in-cell (PIC) simulations [24].

This work studies plasma expansions in magnetic nozzles by using a non-stationary direct Vlasov code. As compared to stationary fluid models, this technique computes the pressure tensor, the heat fluxes and the population of trapped electrons self-consistently. PIC codes do also exhibit these two features. However, due to the numerical noise, they do not give an accurate description if the number of macroparticles per cell is small, a circumstance that is unavoidable in non-stationary simulations of a plasma expansion into vacuum. Direct Vlasov codes, which are more demanding from a computational point of view because they discretize the distribution function in real and velocity spaces instead of using macroparticles, provide a better accuracy and degree of detail of the distribution functions. Section 2 presents the mathematical model and describes briefly the numerical algorithm. The effects of fixing in the code the size of the nozzle (finite simulation domain) and the electrostatic potential value at the exit, two parameters that do not appear or are not externally imposed in a real infinite plasma expansion, are shown in Sec. 3. The correct selection of these two parameters allows to reproduce with the code the conditions of a real nozzle with zero net current. Section 4 shows some kinetic features of the expansion such as the distribution functions, and particle densities, temperature, and heat fluxes. The trapped electron population is computed and the results are compared with previous studies. Section 5 summarizes the conclusions of the work.

\section{Magnetic nozzle model based on guiding center theory}

\subsection{Plasma model}

Let us consider a tank placed at $z<z_{0}<0$ and filled with an electron-ion plasma. We are interested in the time-dependent, magnetically-channeled plasma expansion that is produced when a hole of radius $R_{0}$ at $z=z_{0}$ is opened at the plasma-vacuum wall. For the sake of illustration, the geometry of the magnetic nozzle is the one corresponding to a current loop of radius $R_{L}\left(R_{L}>R_{0}\right)$ placed at the plane $z=0$. It generates a stationary and non-uniform magnetic field in the vacuum region that reaches its maximum value $B_{T}$ at $z=0$ (the nozzle throat $\mathrm{T}$ ). The forward distribution functions of ions and electrons entering the nozzle are assumed semi-Maxwellian,

$$
f_{\alpha}\left(t, z=z_{0}, v_{\|}>0, v_{\perp}\right)=N^{*}\left(\frac{m_{\alpha}}{2 \pi T_{\alpha}^{*}}\right)^{3 / 2} \exp \left(-\frac{m_{\alpha} v^{2}}{2 T_{\alpha}^{*}}\right), \quad \alpha=i, e,(1)
$$

while the backward distribution functions will be determined self-consistently by the expansion characteristics. Here $N^{*}$ and $T_{\alpha}^{*}$ are reference parameters (not the actual densities and temperatures at $z=z_{0}$ that also involve the backward distribution

function), $v=\sqrt{v_{\|}^{2}+v_{\perp}^{2}}$ is the velocity, and $v_{\|}$and $v_{\perp}$ the velocity component parallel and normal to the magnetic field lines. For convenience, hereafter the axial 
coordinate, time, velocities, magnetic field, electrostatic potential, particle distribution functions, and densities, are all normalized and we will write $z / \lambda_{D e}^{*} \rightarrow z, t \omega_{p e}^{*} \rightarrow t$, $v_{\|, \perp} / \lambda_{D e}^{*} \omega_{p e}^{*} \rightarrow v_{\|, \perp}, B / B_{T} \rightarrow B, e \phi / k_{B} T_{e}^{*} \rightarrow \phi, f_{\alpha} / N^{*}\left(m_{e} / k_{B} T_{e}^{*}\right)^{3 / 2} \rightarrow f_{\alpha}$, where $\lambda_{D e}^{*}=\sqrt{\epsilon_{0} k_{B} T_{e}^{*} / N^{*} e^{2}}$ is the Debye length, $\omega_{p e}^{*}=\sqrt{N^{*} e^{2} / m_{e} \epsilon_{0}}$ the electron plasma frequency, $k_{B}$ the Boltzmann constant, $m_{e}$ the electron mass, $e$ the elementary charge, and $\epsilon_{0}$ the vacuum permittivity. As shown below, the plasma dynamics depends on the following dimensionless parameters in our model

$$
r_{L} \equiv \frac{R_{L}}{\lambda_{D e}^{*}}, \quad \delta_{\alpha} \equiv \frac{T_{\alpha}^{*}}{T_{e}^{*}}, \quad \beta_{\alpha} \equiv \frac{m_{\alpha}}{m_{e}}, \quad Z_{\alpha},
$$

where the subscript $\alpha=e, i$ denotes electrons and ions, and $m_{\alpha}$ and $Z_{\alpha}$ are the mass and the charge number of the $\alpha$-species.

In this work we follow a paraxial approximation and assume a slender and slowlyvarying magnetic field, i.e. we take $R_{L} / R_{0}>>1$, and just look at the center line of the magnetic nozzle. Under this hypothesis, the parameter $R_{0}$ does not appear anymore in the model and one just needs the normalized magnetic field at the center line. It reads

$$
\boldsymbol{B}(z)=\frac{r_{L}^{3}}{\left(r_{L}^{2}+z^{2}\right)^{3 / 2}} \mathbf{1}_{z},
$$

where $\mathbf{1}_{z}$ is an unit vector along the $z$-axis. Therefore, we are having a convergentdivergent nozzle with the maximum of the magnetic field $B=1$ at $z=0$ and $B \rightarrow 0$ as $z \rightarrow \pm \infty$; the analysis here will be focussed at the divergent side of the nozzle. The model also assumes that the magnetic field is very strong and the normalized Larmor radii satisfies $\rho_{L \alpha} \equiv \beta v_{\perp} /\left|Z_{\alpha}\right| B<<r_{L}$. In the limit $\rho_{L \alpha} / r_{L} \rightarrow 0$, the slow drift motion of the particles across the field lines can be ignored and the normalized magnetic moment

$$
\mu_{\alpha}=\frac{\beta_{\alpha} v_{\perp}^{2}}{2 B}
$$

is conserved (for brevity, we will generally write $\mu_{\alpha} \rightarrow \mu$ ). Hereafter, we will work with the gyrocenter variables $\left(z, v_{\|}, \mu, \gamma\right)$, and will also average the distribution functions of the particles $f_{\alpha}$ over the fast gyrophase $\gamma$

$$
\bar{f}_{\alpha}\left(t, z, v_{\|} ; \mu\right)=\frac{1}{2 \pi} \int_{0}^{2 \pi} f_{\alpha}\left(t, z, v_{\|}, \mu, \gamma\right) d \gamma .
$$

The evolution of the gyrocenter distribution function $\bar{f}_{\alpha}$ is governed by the Vlasov equation

$$
\frac{\partial \bar{f}_{\alpha}}{\partial t}+v_{\|} \frac{\partial \bar{f}_{\alpha}}{\partial z}+a_{\alpha} \frac{\partial \bar{f}_{\alpha}}{\partial v_{\|}}=0,
$$

where we ignored the induced magnetic field and introduced the parallel dimensionless acceleration

$$
a_{\alpha}=-\frac{1}{\beta_{\alpha}}\left(Z_{\alpha} \frac{\partial \phi(t, z)}{\partial z}+\mu \frac{d B(z)}{d z}\right)
$$


The normalized electric field $\boldsymbol{E}=E_{\|} \boldsymbol{B} / B=-\partial \phi / \partial z$ is given by the paraxial Poisson's equation

$$
B \frac{\partial}{\partial z}\left(\frac{E_{\|}}{B}\right)=\sum_{\alpha=e, i} Z_{\alpha} n_{\alpha}
$$

with the particle densities computed from the distribution functions as

$$
n_{\alpha}(z)=\int f_{\alpha} d \boldsymbol{v}=\frac{2 \pi B}{\beta_{\alpha}} \int_{-\infty}^{+\infty} \int_{0}^{+\infty} \bar{f}_{\alpha} d v_{\|} d \mu .
$$

Therefore, the dynamics of the electrons and the ions governed by the two Vlasov equations in Eq. (6) are nonlinearly coupled through the electrostatic potential. This set of equations must be integrated with appropriate boundary and initial conditions, discussed in Subsec. 2.3.

\subsection{Evolution of macroscopic quantities}

The evolution equations of the main macroscopic quantities are helpful in the analysis of the simulations. The average or mean value of any quantity $\psi$, is computed as

$$
\langle\psi\rangle_{\alpha}=\frac{1}{n_{\alpha}} \int \psi f_{\alpha} d \boldsymbol{v}=\frac{2 \pi B}{\beta_{\alpha} n_{\alpha}} \int_{-\infty}^{+\infty} \int_{0}^{+\infty} \psi \bar{f}_{\alpha} d v_{\|} d \mu
$$

Interesting quantities are: densities $n_{\alpha}=\langle 1\rangle_{\alpha}$, Eq.(9); macroscopic velocities parallel to the magnetic field $u_{\alpha}=\left\langle v_{\|}\right\rangle_{\alpha}$; current densities $j_{\alpha}=Z_{\alpha} n_{\alpha} u_{\alpha}$; temperatures $T_{\| \alpha}=\beta_{\alpha}\left\langle c_{\| \alpha}^{2}\right\rangle_{\alpha}$ and $T_{\perp \alpha}=B\langle\mu\rangle_{\alpha}$, where we introduced the peculiar velocities $c_{\| \alpha}=v_{\|}-u_{\alpha} ;$ pressures $P_{\| \alpha}=n_{\alpha} T_{\| \alpha}$ and $P_{\perp \alpha}=n_{\alpha} T_{\perp \alpha}$; and (parallel) heat fluxes of parallel and perpendicular energy, $Q_{\| \alpha}=\frac{1}{2} \beta_{\alpha} n_{\alpha}\left\langle c_{\| \alpha}^{3}\right\rangle_{\alpha}$ and $Q_{\perp \alpha}=B n_{\alpha}\left\langle\mu c_{\| \alpha}\right\rangle_{\alpha}$, respectively. According to Sec. 2, the normalization has been done with characteristic variables involving the electron mass.

The evolution equations of these quantities are obtained straightforwardly by taking velocity moments in Eq. (6). In the paraxial case, the equations for continuity, axial momentum, total energy, and perpendicular energy are, respectively,

$$
\begin{aligned}
& \frac{\partial n_{\alpha}}{\partial t}+B \frac{\partial}{\partial z}\left(\frac{n_{\alpha} u_{\alpha}}{B}\right)=0 \\
& \frac{\partial}{\partial t}\left(\beta_{\alpha} n_{\alpha} u_{\alpha}\right)+B \frac{\partial}{\partial z}\left(\frac{\beta_{\alpha} n_{\alpha} u_{\alpha}^{2}}{B}\right)=-Z_{\alpha} n_{\alpha} \frac{\partial \phi}{\partial z} \\
& \quad+\left[\left(P_{\| \alpha}-P_{\perp \alpha}\right) \frac{\partial \ln B}{\partial z}-\frac{\partial P_{\| \alpha}}{\partial z}\right], \\
& \frac{\partial}{\partial t}\left[n_{\alpha}\left(\frac{\beta_{\alpha}}{2} u_{\alpha}^{2}+\frac{T_{\| \alpha}}{2}+T_{\perp \alpha}\right)\right]+ \\
& B \frac{\partial}{\partial z}\left[\frac{n_{\alpha} u_{\alpha}}{B}\left(\frac{\beta_{\alpha}}{2} u_{\alpha}^{2}+\frac{3}{2} T_{\| \alpha}+T_{\perp \alpha}\right)+\frac{Q_{\| \alpha}+Q_{\perp \alpha}}{B}\right]+j_{\alpha} \frac{\partial \phi}{\partial z}=0 . \\
& \frac{\partial}{\partial t}\left(n_{\alpha} T_{\perp \alpha}\right)+B^{2} \frac{\partial}{\partial z}\left[\frac{1}{B^{2}}\left(n_{\alpha} u_{\alpha} T_{\perp \alpha}+Q_{\perp \alpha}\right)\right]=0,
\end{aligned}
$$


This set of macroscopic equations is incomplete, unless equations for the parallel heat fluxes $Q_{\| \alpha}$ and $Q_{\perp \alpha}$ are added, which will introduce higher order magnitudes. A closure of the set of the fluid equations is not simple in a collisionless plasma.

Here, the consistent kinetic solution is obtained directly, so the fluid equations are used to interpret the results, mainly the steady-state ones in Section 3. In this respect, in the above equations, $1 / B$ plays the role of the effective beam area [furthermore, it is in fact the natural spatial variable (instead of $z$ ) in the divergent paraxial nozzle]. Thus, in (11), $n_{\alpha} u_{\alpha} / B$ is the species flow (i.e. the flux area integrated), which is constant spatially in steady-state. The species current, $j_{\alpha} / B$, and the total plasma current, $I=\left(j_{e}+j_{i}\right) / B$, are constant in steady-state too. In (12), the two last terms on the right-hand side are the contribution of the divergence of the pressure tensor (i.e. the net pressure force). Then, the steady-state limit of (13) yields that the total enthalpy flow, $\dot{H}_{\alpha}$, is constant spatially,

$$
\dot{H}_{\alpha} \equiv \frac{n_{\alpha} u_{\alpha}}{B}\left(\frac{\beta_{\alpha}}{2} u_{\alpha}^{2}+\frac{3}{2} T_{\| \alpha}+T_{\perp \alpha}+Z_{\alpha} \phi\right)+\frac{Q_{\| \alpha}+Q_{\perp \alpha}}{B}=\text { const }
$$

Here, $\left(Q_{\| \alpha}+Q_{\perp \alpha}\right) / B$ is the total heat conduction flow. The steady state limit of (14) yields that the (convection plus conduction) flow of perpendicular energy evolves proportional to $B^{-1}$

$$
\frac{n_{\alpha} u_{\alpha} T_{\perp \alpha}+Q_{\perp \alpha}}{B^{2}}=\text { const }
$$

which is the direct consequence of the conservation of the magnetic moment of the species. These conservation laws were already used in Ref. [25] to analyze the plasma response in a convergent magnetic field. Finally, if the mean kinetic energy is eliminated from (13) by using equations (11) and (12), the evolution equation for the internal energy is obtained,

$$
\begin{gathered}
\frac{\partial}{\partial t}\left(\frac{P_{\| \alpha}}{2}+P_{\perp \alpha}\right)+B \frac{\partial}{\partial z}\left\{\frac{1}{B}\left[u_{\alpha}\left(\frac{3}{2} P_{\| \alpha}+P_{\perp \alpha}\right)+\left(Q_{\| \alpha}+Q_{\perp \alpha}\right)\right]\right\} \\
-u_{\alpha}\left[\frac{\partial P_{\| \alpha}}{\partial z}+\left(P_{\perp \alpha}-P_{\| \alpha}\right) \frac{\partial \ln B}{\partial z}\right]=0
\end{gathered}
$$

which can susbtitute for (13).

\subsection{Simulation domain and boundary conditions}

We are interested in the time-dependent plasma expansion along the (semi-infinite) divergent nozzle, extending from $z=0$ (the throat $T$ ) to $z=\infty$. However, since the numerical simulation requires to work with a finite domain, the downstream end of the domain (point M) will be placed at a certain $z_{M} \gg 1$, with $B_{M}<<1$. A parametric analysis of the combined influence of $z_{M}$ and $r_{L}$ on the solution is carried out below. Furthermore, it turns out that, in spite of applying quasineutrality at the upstream end of the simulation domain, a non-desirable Debye sheath, extending a few Debye lengths develops there. In order to eliminate its spurious influence, the usptream end 
of the domain has been placed at the convergent side of the nozzle, in particular, at $z_{0}=-r_{L} / 2$.

At the domain entrance, we set Maxwellian functions for the injected particles,

$$
\bar{f}_{\alpha}\left(t, z=z_{0}, v_{\|}>0 ; \mu\right)=\chi_{\alpha}(t) \bar{f}_{M \alpha}
$$

with

$$
\bar{f}_{M \alpha}=\left(\frac{\beta_{\alpha}}{2 \pi \delta_{\alpha}}\right)^{3 / 2} \exp \left(-\frac{\beta_{\alpha} v_{\|}^{2}}{2 \delta_{\alpha}}-\frac{B \mu}{\delta_{\alpha}}\right)
$$

and $\chi_{e}=1$. The parameter $\chi_{i}(t)$ is dynamically varied to accomplish quasineutrality at entrance section $z=z_{0}$, once reflected-back particles are taken into account there. (For instance, if no ions are reflected back and all electrons are, the $\chi_{i}=2$.) At the domain downstream end, in order to simulate the vacuum at infinity, we impose no incoming particles into the domain,

$$
\bar{f}_{\alpha}\left(t, z=z_{M}, v_{\|}<0 ; \mu\right)=0 .
$$

Regarding initial conditions, one would initially set $\bar{f}_{\alpha}\left(t=0, z>z_{0}, v_{\|} ; \mu\right)=0$. However, since such a hard transition can lead to numerical issues, our simulations used

$$
\bar{f}_{\alpha}\left(t=0, z>z_{0}, v_{\|} ; \mu\right)=\bar{f}_{M \alpha} \times \exp \left(-\frac{z-z_{0}}{L_{0}}\right)
$$

with $L_{0}$ a dimensionless parameter that controls the density gradient of the initial plasma profile. A value $L_{0}=2$, which yields a profile with width about a few Debye lengths, is enough to provide a smooth transition at $t=0$ in the simulations.

Finally, Poisson's equation requires two boundary conditions on the electrostatic potential. Clearly, there is the freedom to take $\phi\left(z=z_{0}\right)=0$. With respect to the boundary condition at the downstream end, the studies of the semi-infinite, stationary nozzle with a simple plasma have shown two things. First, the potential decays monotonically to an asymptotic value $\phi=\phi_{\infty}<0$ (i.e. yielding $d \phi /\left.d z\right|_{\infty} \rightarrow 0$ ). Second, the net electric current of the plasma beam $I$ is not a parameter independent of $\phi_{\infty}$ : a parametric current-voltage curve $I\left(\phi_{M}\right)$ with $\partial I / \partial\left|\phi_{\infty}\right|>0$ exists. This behavior of the current-voltage curve is simple to explain: for normalized distribution functions at injection, the more negative is $\phi_{\infty}$, a larger fraction of electrons injected into the nozzle is reflected back to the reservoir while (near) all ions cross freely the nozzle, and thus the more positive becomes $I$. Therefore, in the downstream end of our finite simulation box we can impose either $\phi_{M}=\phi\left(z_{M}\right)$ or $I$. The first choice is the natural one for the numerical scheme. Notice then, that the case of most practical interest, $I=0$, which corresponds to a current-free plasma beam, requires to iterate on $\phi_{M}$.

\subsection{Direct Vlasov solver}

This section discusses briefly the main features of the novel direct Vlasov code VLASMAN (VLAsov Simulator for MAgnetic Nozzle), that has been developed for the numerical integration of (6). A mesh of points $z_{i}$ with $i=1, \ldots N_{z}$ is defined within the interval $z_{0} \leq z \leq z_{M}$. These points are distributed non-uniformly in order to keep 
constant the ratio between the resolution of the mesh and the local Debye length, which is expected to vary as $\lambda_{D e} \sim n^{-1 / 2} \sim B^{-1 / 2}$. The velocity space, involving $v_{\|}$and $\mu$, was truncated as $-v_{\text {max }}^{\alpha} \leq v_{\|} \leq v_{\text {max }}^{\alpha}$ and $0 \leq \mu \leq \mu_{\text {max }}^{\alpha}$ and discretized with $N_{v_{\|}} \times N_{\mu}$ points. Unlike the spatial mesh, which is common for both species, different maximum velocities and magnetic moments are chosen for electrons and ions. For both species, the velocity mesh is uniform. The unknowns of the code are the values of the distribution functions at the points of the mesh and at discrete times $t_{m}, \bar{f}_{\alpha}\left(t_{m}, s_{i}, v_{\| j}, \mu_{k}\right)$.

Since $\mu$ appears as a parameter in (6), the algorithm just needs to solve a one dimensional equation $N_{\mu}$ times. Given the distribution function $\bar{f}_{\alpha}\left(t_{m}, s_{i}, v_{\| j}, \mu_{k}\right)$, the value at $t_{m}+\Delta t$ is found by using a splitting algorithm that treats the convective terms in the $z$ and $v_{\|}$directions separately, and gives a scheme of second order in $\Delta t$ [26]. A short summary of the splitting algorithm and a description of the numerical schemes implemented for the interpolation and the numerical integration are given in Appendix A.

In the simulations, we took the physical parameters $\delta_{i}=1, \beta_{i}=100, Z_{i}=1$ and considered several values of $r_{L}$. The value of $\beta_{i}$ is not realistic for an electronion plasma but it still separates significantly the electron and ion response times and helped us save computational resources. Regarding the geometry of the nozzle, we set $z_{0}=-r_{L} / 2$, i.e. the divergent and convergent segments have lengths equal to $r_{L} / 2$ and $z_{M}$, respectively. The most relevant numerical parameters are $N_{v_{\|}}=77, N_{\mu}=101$, $v_{\max }^{e}=5, v_{\max }^{i}=0.5, \mu_{\max }^{e}=\mu_{\max }^{i}=12.5$, and $\Delta t=0.03$. Tradeoffs analysis varying the numerical parameters $z_{M}$ and $\phi_{M}$ are shown in Sec. 3.

\section{Stationary solution and parametric analysis}

The effect of the truncation of the computational box up to a length $z_{M}$ and the setting of the electrostatic potential value $\phi_{M}$ at that position have been investigated by running a large number of simulations. After taking an expansion rate of $r_{L}=50$, the physical and numerical parameters explained in Sec. 2.4, and several values of $z_{M}$ and $\phi_{M}$, we integrate the Vlasov-Poisson system forward in time until the plasma reached the corresponding stationary state. For each simulation, the latter was verified by monitoring the time evolution of the most important variables, such as density and potential, and the $z$-profiles of $n_{\alpha} u_{\alpha} / B$, which becomes uniform at stationary conditions.

First of all, Figure 1 shows the results of investigating the influence of the total potential fall $\phi_{M}$ on the electric current $I$ across the nozzle in steady-state. As expected and known from previous models, the current $I$ (abcissa) is positive for large, negative values of $\phi_{M}$ (ordinate), and negative otherwise. The most relevant result here is the universal character of the curve $\phi_{M}(I)$ : it is practically independent of $r_{L}$ and $z_{M}$ (as long as $B_{M} \ll 1$ ), which allows to infer that this curve reproduces the behavior of the semi-infinite nozzle too. Furthermore, Fig. 1 shows that the curve $\phi_{T M}(I)$ for the potential fall along the divergent part of the nozzle is (near) universal too. The principal, current-free beam case has potential falls of $\phi_{M} \approx-2.75$ and $\phi_{T M} \approx-2.34$. 


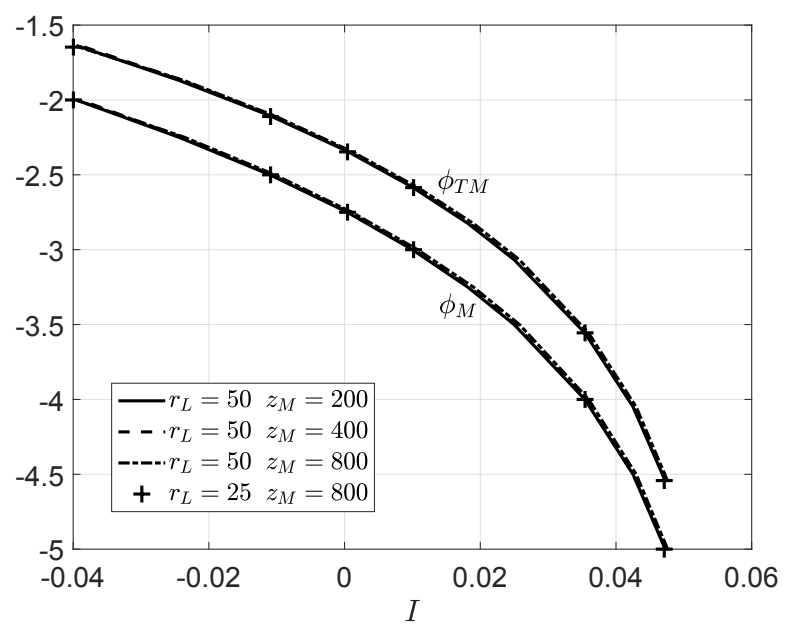

Figure 1. Net current-to-magnetic field ratio $j / B$ versus the total potential drop $\left(\phi_{M}\right)$ and the one between the throat and $z_{M}\left(\phi_{T M}\right)$ for several expansion rates and box sizes. The curves practically overlap, also for the case $r_{L}=100$ (not shown)

Such potential drops are consistent with previous calculations from stationary kinetic models (extrapolate to $m_{i} / m_{e}=100$ the results in Fig. 4c of Ref. [19]). Hereafter, we focus the analysis at discussing the (approximate) 'current-free case' $\phi_{M}=-2.75$. The steady states values of the normalized species current are $I_{i}=-I_{e} \simeq 0.074$
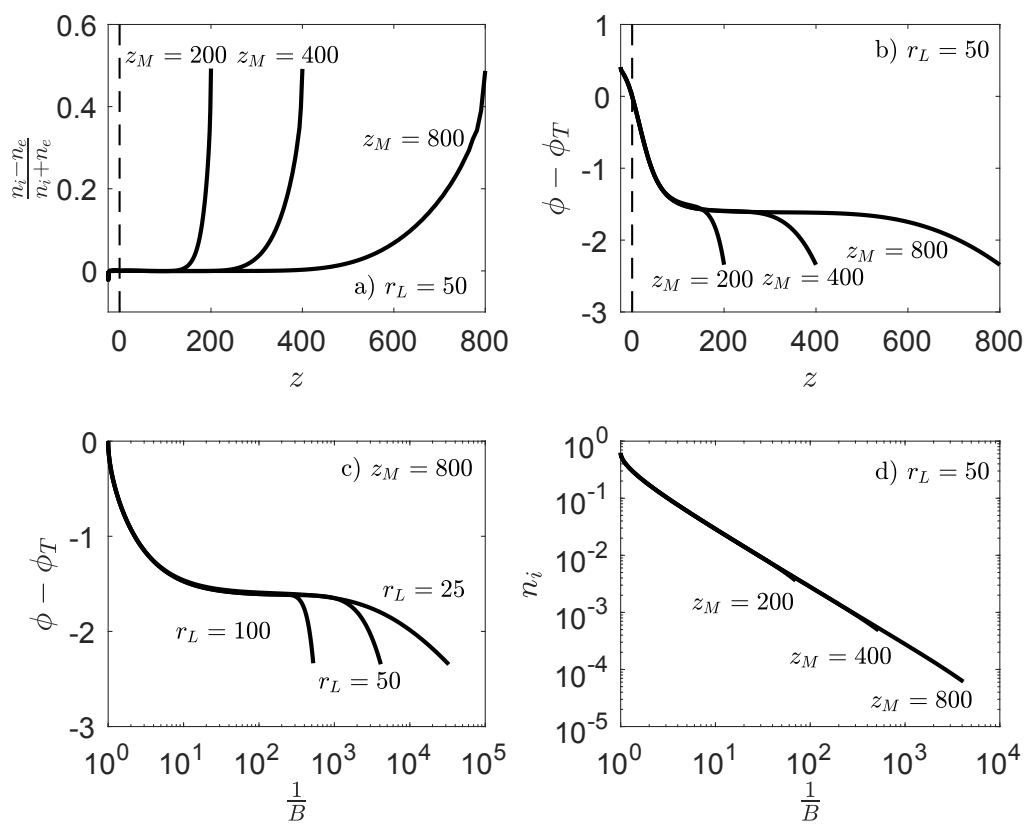

Figure 2. Panels (a), (b) and (d) show, respectively, the normalized space charge, $\phi$ versus $z$ and $n_{i}$ versus $1 / B$ for $r_{L}=50$ and several $z_{M}$. Panel (c) displays $\phi$ versus $1 / B$ for $z_{M}=800$ and several expansion rates. 
Figure 2 shows (near) stationary axial profiles for different $r_{L}$ and $z_{M}$. Panel (a) plots the relative space charge for $r_{L}=50$ and several lengths of the simulation box; panels (b) and (c) plot the electric potential profile versus $z$ and $B^{-1}$, and panel (d) the ion density versus $B^{-1}$. Three different spatial regions can be distinguished in panels (a)-(c). First, a small sheath (with a relative space charge $<5 \%$ and extending a few Debye lengths) forms at the entrance of the simulation domain, in spite of having forced quasineutrality locally at $z=z_{0}$. This 'numerical' sheath is caused by the need of the electric field to adapt the entrance distribution functions of ions and electrons and it was the reason to include a small convergent part of the nozzle, even though the work is focused on the divergent nozzle behavior. Second, there is the large quasineutral region, with a decreasing electric field as we move downstream. Third, there is a second Debye sheath at the downstream end of the simulation. Since the Debye length is proportional to $n_{\alpha}^{-1 / 2}$ [and thus nearly proportional to $B^{-1 / 2}$, according to panel (d)], it increases by 1-2 orders of magnitude along the discharge, thus giving the impression that the downstream sheath is thick. Just for reporting, the relative space-charge and the potential fall in the downstream sheath are nearly constant, because of the low electric field at the sheath entrance and the need to adjust the total potential fall to $\phi_{M}$. Panel (c) shows that $\phi$ depends more naturally on $B^{-1}$ in the quasineutral region. In panel (d), we see that $n_{i}$ is near proportional to $B$, indicating a much gentler dependence of $u_{i}$ on $B$ (to have $n_{i} u_{i} / B=$ const). The electron density $n_{e}$ behaves as $n_{i}$ except at the downstream sheath where it decreases more sharply. Normalized plasma densities at the entrance and the throat are $n_{e 0}=0.97$ and $n_{e T}=0.62$, the difference indicating the jet acceleration in the small convergent region of the nozzle. Finally, at the simulation box entrance, the ratio of ion-to-electron densities for forward moving particles, that is $\chi_{i}$ in (18) is 1.53: this ratio would be close to 2 and 1 if the entrance would be, respectively, at the throat and further upstream in the convergent magnetic nozzle.
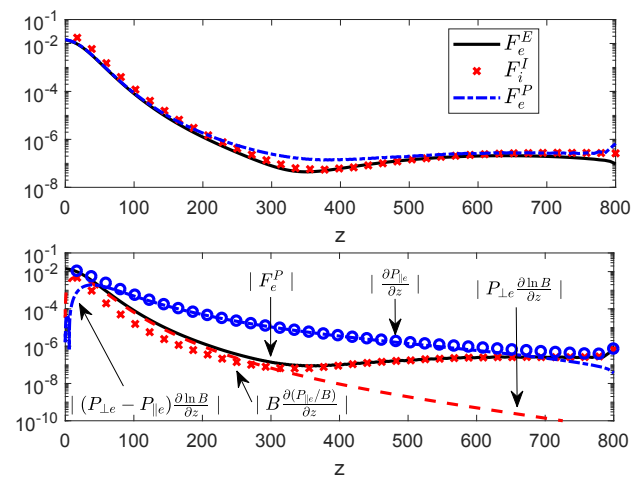

Figure 3. Stationary, spatial profiles of inertial, pressure, and electric forces of ions and electrons for $r_{L}=50$ and $z_{M}=800$.

The stationary momentum equation of ions and electrons, (12), states the balance

$$
F_{\alpha}^{I}=F_{\alpha}^{P}+F_{\alpha}^{E}
$$


between the inertial force (or flow of species momentum) $F_{\alpha}^{I}$, the pressure force $F_{\alpha}^{P}$, and the electric force $F_{\alpha}^{E}$. Both $F_{\alpha}^{I}$ are positive, both $F_{\alpha}^{P}$ are expected to be positive, while $F_{i}^{E}>0$ and $F_{e}^{E}<0$. Furthermore, one has: $F_{e}^{E} \simeq F_{i}^{E}$ in the quasineutral region; and $F_{e}^{I} / F_{i}^{I} \sim m_{e} / m_{i} \ll 1$ for the current-free and 'small current' cases. The combination of these two facts implies that the electron inertia is negligible, and the electric and electron pressure forces balance each other, i.e. $F_{e}^{P} \simeq-F_{e}^{E} \gg F_{e}^{I}$. For ions, the inertial force is dominant, and the relevance of the ion pressure depends on upstream conditions for $T_{i} / T_{e}$. These trends are confirmed by Figure 3(a), which plots $F_{e}^{E}, F_{i}^{E}, F_{e}^{P}$, and $F_{i}^{I}$; the two other forces are obtained by just applying (22). In this case the ion pressure is rather small (since $T_{i}^{*} \ll \phi_{M}, T_{e}^{*}$ ) so that the ion flow is accelerated freely by the electric force.

Figure 3(b) assesses the different contribution to the net electron pressure force, that is the pressure tensor divergence. This can be expressed in two different ways:

$$
\nabla \cdot \overline{\bar{P}}_{\alpha}=\frac{\partial P_{\| \alpha}}{\partial z}+\left(P_{\perp \alpha}-P_{\| \alpha}\right) \frac{\partial \ln B}{\partial z} \equiv B \frac{\partial}{\partial z}\left(\frac{P_{\| \alpha}}{B}\right)+P_{\perp \alpha} \frac{\partial \ln B}{\partial z} .
$$

The first division is based on the parallel pressure gradient and the magnetic mirror effect, and the second is based on the $P_{\| \alpha}$ and $P_{\perp \alpha}$ contributions. The panel shows, interestingly, that the parallel pressure gradient and the magnetic mirror effect are individually much larger as their difference (i.e. they compensate practically each other). This makes them not very suitable to characterize the total pressure contribution. On the contrary the $P_{\| \alpha}$ and $P_{\perp \alpha}$ contributions are of the same order than their difference. For this particular case, the $P_{\perp \alpha^{-}}$-contribution dominates mildly upstream, while the $P_{\| \alpha^{-}}$ contribution dominates totally downstream. The milder behavior of the $z$-derivative of $P_{\| \alpha} / B$ compared to that of $P_{\| \alpha}$, and the drop of the $P_{\perp \alpha}$-contribution, are due to $n_{\alpha} \propto B$ approximately and the behaviors of $T_{\| \alpha}$ and $T_{\perp \alpha}$ shown below. Although not shown, the ion pressure contributions behave in the same way than the electron ones.

Figure 4(a)-(c) displays the stationary spatial profiles of the parallel and perpendicular temperatures of ions and electrons for $z_{M}=800$ and $r_{L}=25,50$, and 100 . In this collisionless plasma, these kinetic temperatures simply express the dispersion of particle velocities. The first interesting feature is that both perpendicular temperatures decrease with $B^{-1}$, a behavior related to the conservation of magnetic moment. On the contrary, the parallel temperatures are rather constant spatially, except for the decrease of $T_{\| e}$ at the downstream sheath. Therefore, the plasma expansion along the divergent nozzle implies both anisotropy and cooling [the average temperature is $\left(T_{\| \alpha}+2 T_{\perp \alpha}\right) / 3$ ] This behavior agrees qualitatively with stationary, kinetic, fully-quasineutral models (see figure 7 in [19]). Apparently, the main difference of the non-stationary model, which computes the population of trapped electrons self-consistently, is a softer decay of the parallel temperature. Values of normalized temperatures at the throat are $T_{\| i T} \simeq 0.30$, $T_{\perp i T} \simeq 1.20, T_{\| e T} \simeq 0.86, T_{\perp e T} \simeq 0.90$, and they are explained by the analysis of Ref.[25] for a convergent magnetic geometry: there the ion distribution function is determined by the combination of the magnetic mirror and the free extraction at the throat, while electrons remain near Maxwellian. Figure 4(d) displays the ion axial kinetic energy, 

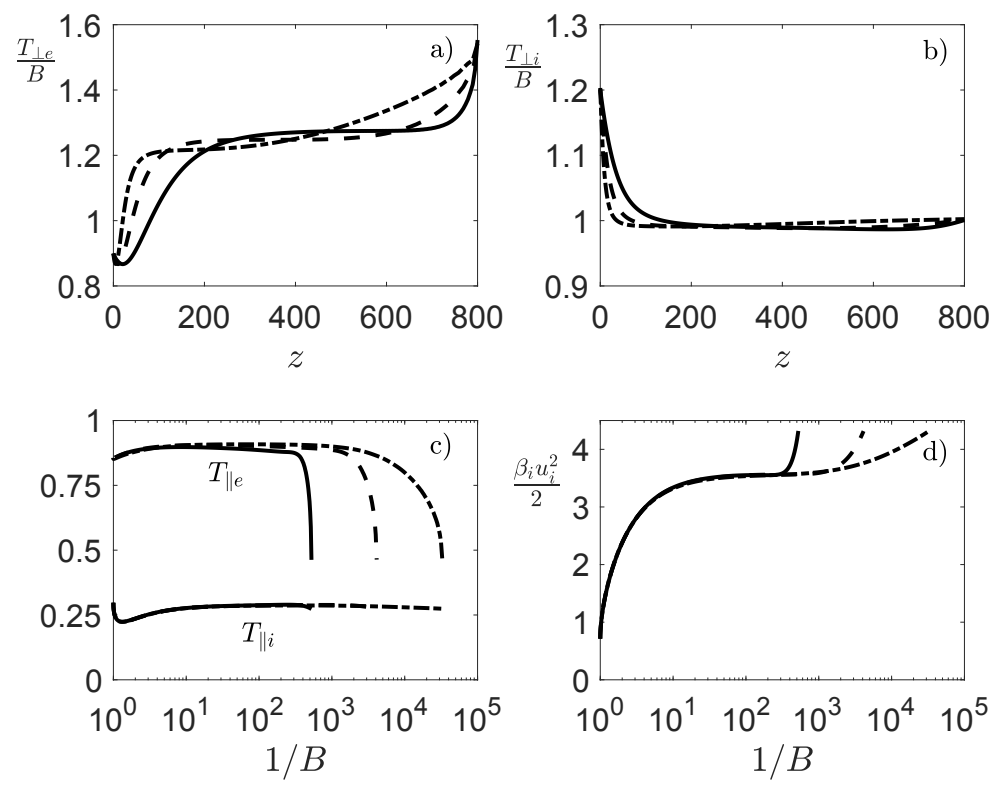

Figure 4. Panels (a) and (b) show the perpendicular temperature of the electron and the ions, respectively.Panel (c) shows their parallel temperatures and (d) the mean kinetic energy of the ions. $z_{M}=800$ and solid, dashed, and dot-dash lines correspond to $r_{L}=100,50$, and 25 , respectively.

which increases downstream thanks to the electric potential energy. For this currentfree case, the electron axial energy behaves exactly the same in the quasineutral region (but it is $m_{e} / m_{i}$ times lower).
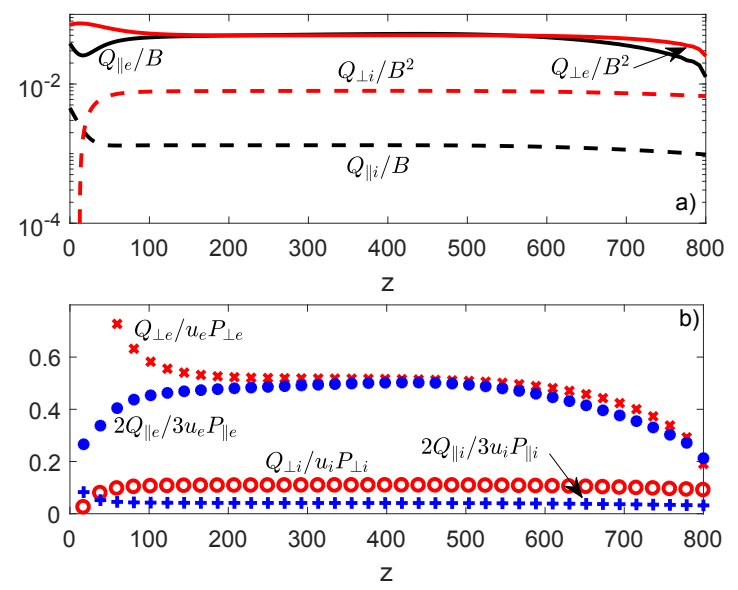

Figure 5. Axial profiles of the heat fluxes [panel (a)] and their relative rates versus the pressure fluxes [panel (b)] $r_{L}=50$ and $z_{M}=800$.

Figure 5 analyzes the $z$-profiles of the heat (or internal energy conduction) flows. We recall that $Q_{\|}$and $Q_{\perp}$ represent parallel fluxes of the parallel and perpendicular 
thermal energies (perpendicular fluxes are zero in our model). Notice that, since this is a collisionless plasma, no Fourier-type law is expected to apply for these heat flows. Panel (a) shows that: the area-integrated parallel heat flows, $Q_{\| \alpha} / B$, are approximately constant (except, as often, near the ends of the simulations) while the parallel flow of perpendicular thermal energy, $Q_{\perp \alpha} / B$, decrease proportional to $B^{2}$. The same dependence with $B$ was found for the internal energy convection flows, $n_{\alpha} u_{\alpha}\left(3 T_{\| \alpha} / 2\right)$ and $n_{\alpha} u_{\alpha} T_{\perp \alpha}$. To complete this, panel (b) determines the relative rates of internal energy conduction versus the pressure flux for ions and electrons. Focusing on the main quasineutral region, conduction of parallel and perpendicular internal energies of electrons is about 50-52\% of its convection; for ions conduction represents $4 \%$ and $11 \%$ in the parallel and perpendicular cases; more parametric analyses are needed to ascertain how these values depend on the upstream plasma conditions. In spite of this, it seems clear that, both for ions and electrons, the internal energy perpendicular flow decreases in proportion to $B$, Eq. (16), and becomes negligible downstream. Then, since the total enthalpy flow is conserved, Eq. law (15), that loss of perpendicular energy goes mainly into increasing the axial kinetic energy, which is the macroscopic equivalence of the classical particle mirror effect, for ions, and the electric potential energy for electrons.

\section{Kinetic features of the expansion}

There are several ways of presenting the four-dimensional distribution functions $f_{\alpha}\left(t, z, v_{\|}, \mu\right)$ and each of them highlights a particular physical feature of the plasma expansion. For instance, as shown in Figs. 6 and 7, the development of the plasma plume structure is evident when $f_{\alpha}$ is plotted versus the axial distance and the parallel velocity for a given value of $\mu$ and $t$. In panels (a)-(c) of Fig. 6, which corresponds to that representation with $\mu_{e}=1$ and $t=500,2000$, and 7200 for $r_{L}=50$ and $z_{M}=800$, one can observe how the simulation box is filled progressively by the plasma. Note that our simulations used $v_{\max }^{e}=5$ but we only showed the range $-3 \leq v_{\| e} \leq 3$ for clarity. As shown by the red line, the distribution $f_{e}$ is not symmetric with respect to the axis $v_{\| e}=0$; for a given $z$, there are more outgoing electrons than reflected ones. As shown in panel (c), the depletion of particles with negative velocity as $z$ increases gives rise to a lower dispersion in $v_{\| e}$, thus producing the moderate drop in $T_{\| e}$ displayed in figure 4 .

The distribution functions of ions with magnetic moment $\mu_{i}=1$ and times $t=500$, 2000 and 7200 are shown in figure 7. For convenience, we did not plot the full dynamic range of the simulation $\left(-0.5 \leq v_{\| e} \leq 0.5\right)$ but only the region of interest. Panels (a)-(c) reveal that ions reach higher velocities during the transient than in stationary conditions. Once the plume reached its equilibrium state [panel (c)], most of the acceleration happens within the first sixty Debye lengths from the throat [consistently with the electrostatic potential profile of panel (c) in Fig. 2]. Although there is a certain amount of dispersion, the ion distribution function is not far from mono-energetic. 


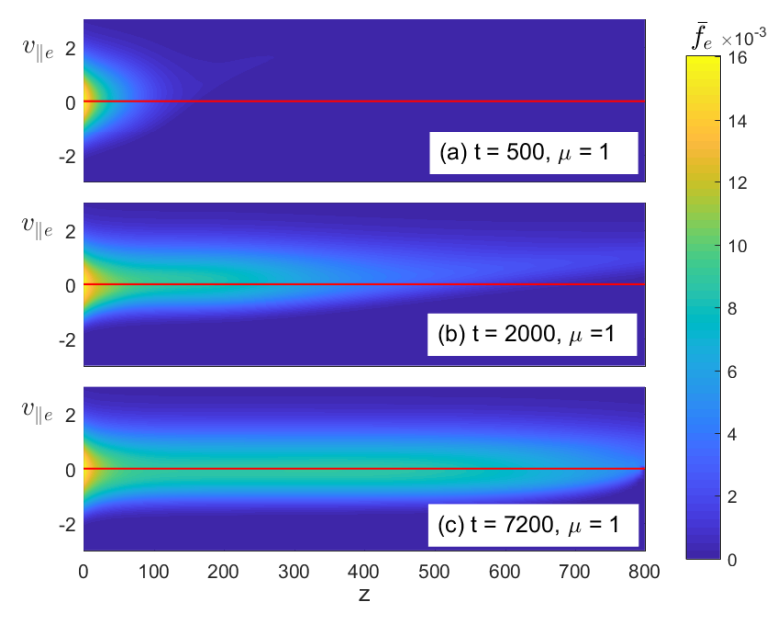

Figure 6. Panels (a), (b), and (c) show the electron distribution function for $\mu=1$ and times $t=500,2000$ and 7200, respectively, for $r_{L}=50$ and $z_{M}=800$. The red line corresponds to $v_{\| e}=0$.

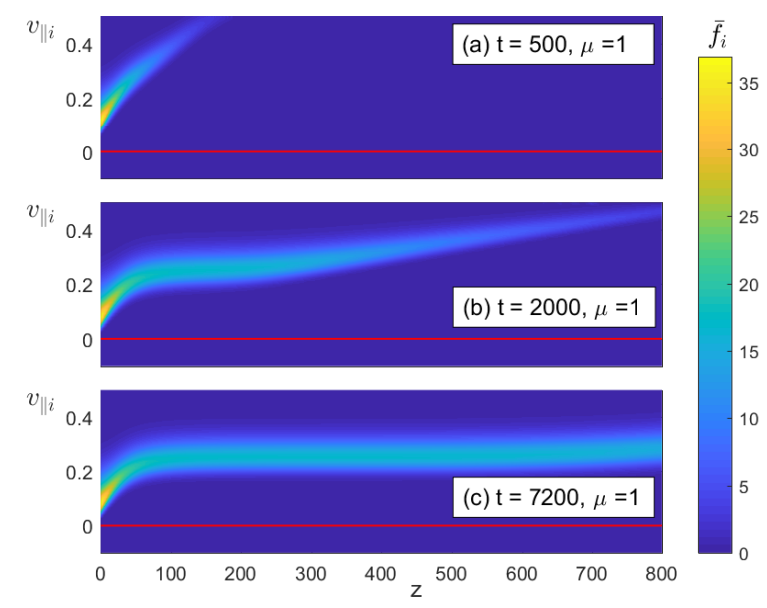

Figure 7. Panels (a), (b), and (c) show the ion distribution function for $\mu=1$ and times $t=500,2000$ and 7200, respectively, for $r_{L}=50$ and $z_{M}=800$. The red line corresponds to $v_{\| i}=0$.

\subsection{Trapped electrons}

One of the main features that distinguishes the non-stationary code VLASMAN from other codes is the self-consistent computation of the doubly-trapped particles (electrons, here). A detailed analysis of this population is carried out by noting first that the characteristic equations of the Vlasov equation

$$
\begin{aligned}
& \frac{d z}{d t}=v_{\|}, \\
& \frac{d v_{\|}}{d t}=a_{\alpha}(z, t, \mu),
\end{aligned}
$$


give the following evolution law for the particle total energy $E=\beta_{\alpha} v_{\|}^{2} / 2+Z_{\alpha} \phi+\mu B$

$$
\frac{d E}{d t}=Z_{\alpha} \frac{\partial \phi}{\partial t} .
$$

Therefore, in stationary conditions $(\partial \phi / \partial t=0)$, the energy is also conserved. In the steady state and for a given energy and $z$ position, the maximum value of the magnetic moment

$$
\mu_{\max }(z, E)=\frac{E-Z_{\alpha} \phi}{B}
$$

is found by setting $v_{\|}=0$ in the definition of the energy. A particle of energy $E$ is trapped between two axial coordinates $z_{\min } \leq z \leq z_{\max }$ if its magnetic moment $\mu$ intersects the curve of $\mu_{\max }$ at those points, i.e. $\mu_{\max }\left(z_{\min }, E\right)=\mu_{\max }\left(z_{\max }, E\right)=\mu$. For this reason, the analysis of the trapped particles is easier with the parametrization $f_{e}(t, z, E, \mu)$, instead of $f_{e}\left(t, z, v_{\|}, \mu\right)$. Since for a given energy there are two possible velocities $v_{\|}= \pm \sqrt{2\left(E-Z_{\alpha} \phi-\mu B\right) / \beta_{\alpha}}$, the total distribution function $f_{e}(t, z, E, \mu)$ involves outgoing $f_{e}^{+}\left(v_{\|}>0\right)$ and ingoing $f_{e}^{-}\left(v_{\|}<0\right)$ distribution functions. In the analysis below we will always refer to the total one.

Panels (a)-(c) in Fig. 8 show the electron distribution function versus $z$ and $\mu$ for $E=2.1$ and times $t=500,2000$ and 7200. At large $t$ [panel (c), considered as steady state], the curve $\mu_{\max }$ (upper red solid line) exhibits a minimum, say $\mu^{*}$, at a position $z^{*}$ close to the throat. For this energy level, two populations of electrons exist in the steady state. First, reflected electrons, with $\mu<\mu^{*}$ or $\mu>\mu^{*}$ and $z<z^{*}$ : they are injected from the left of the simulation box, propagate to the right until the location where $\mu=\mu_{\max }$, where $v_{\|}=0$, and they are reflected back to the nozzle entrance and the reservoir. Second, trapped electrons, with $\mu>\mu^{*}$ and $z>z^{*}$, which bounce between two axial coordinates satisfying $\mu_{\max }\left(z_{\min }, E\right)=\mu_{\max }\left(z_{\max }, E\right)=\mu$. Physically, in the divergent nozzle, electrons injected upstream gain parallel velocity from the (anti) mirror effect in the decaying $B$ and lose it from the electrostatic force. Therefore, their reflection is always due to the dominance of the electrostatic force. The same type of arguments apply to the bouncing motion of the double-trapped electrons.

Similarly to panel (c), panel (d) shows the steady-state distribution function for electrons of a higher energy $(E=3), \mu_{\max }\left(z_{\max }\right)>0$. In this case, there is a population of free electrons with $\mu<\mu^{*}$ that leave the computational box together with the ions, there is a small region of reflected electrons, there are no doubly trapped electrons, and the region $\mu>\mu^{*}$ and $z>z^{*}$ will be totally void of electrons at $t \rightarrow \infty$. Hereafter we will denote the doubly-trapped, reflected and free electrons with the subscripts et, er, and $e f$, respectively.

The filling of the regions of the phase space with trapped particles, which is not connected with the injection zone $(z=0)$ at stationary conditions, is explained in terms of two transient mechanisms. Since the curve $\mu_{\max }$ and its minimum $\mu^{*}$ depend on time, an electron moving to the right with $\mu<\mu^{*}$ at a given time could be trapped if it meets the condition $\mu>\mu^{*}$ when moving backwards after electrostatic reflection. The other mechanism cannot be visualized in the $\mu-z$ plane because it is related to the transport 
of particles along a third dimension (the energy is not conserved during the transient). A particle, with energy $E_{j}$ at instant $t_{j}$ and non-trapped in the $\mu-z$ diagram with $E=E_{j}$, could be trapped at a latter instant $t_{k}$ if its energy decreases to a value $E_{k}$ that traps the particle at the $\mu-z$ diagram with $E=E_{k}<E_{j}$. Both mechanisms, which can trap or untrap the particles, act simultaneously during the transient period of the simulation.

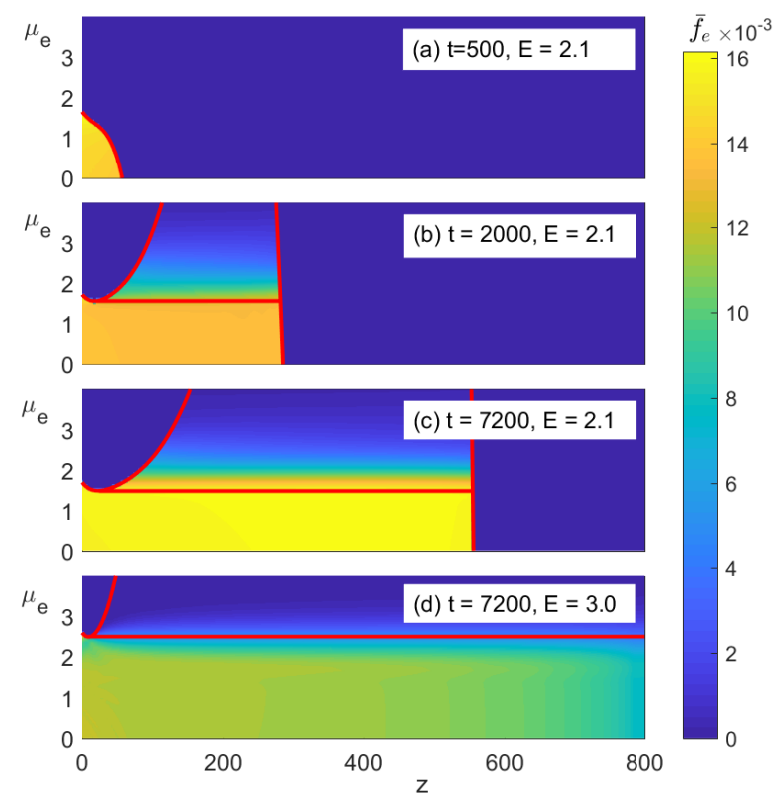

Figure 8. Electron distribution functions in the $\mu_{e}-z$ plane for several values of $E$ and $t$. Simulations results for $r_{L}=50$ and $z_{M}=800$. The red line corresponds to $\mu_{\max }$ in equation 27 and $\mu=\mu^{*}$.

In order to assess the relative importance of the three populations of electrons we analyzed the distribution function at the end of the simulations with $r_{L}=25,50$, and 100. From the $z-\mu$ diagrams for all the energies involved in the simulations, the densities of the trapped $\left(n_{e t}\right)$, free $\left(n_{e f}\right)$, and reflected $\left(n_{e r}\right)$ electrons were found. The results are displayed in the three panels of Fig. 9, which show the densities versus the inverse of the magnetic field. In the extensive quasineutral region the three densities are of the same order and the trapped electrons represent about $20 \%$ of the total. Such a value shows that the population of trapped electrons is neither dominant nor negligible. In any case, it is smaller than the one assumed heuristically by a recent stationary kinetic model, which filled completely the phase-space region where doubly-trapped electrons could potentially exist [19]. At the downstream sheath, where only the most energetic electrons can arrive, the density of the free electrons is clearly dominant but the density of trapped electrons still has a finite value.

The results of the code Vlasman can be post-processed to investigate more advanced features related with the trapping of the particles. For instance, once a simulation is finished and the history of the electrostatic potential $\phi(z, t)$ and the distribution function 

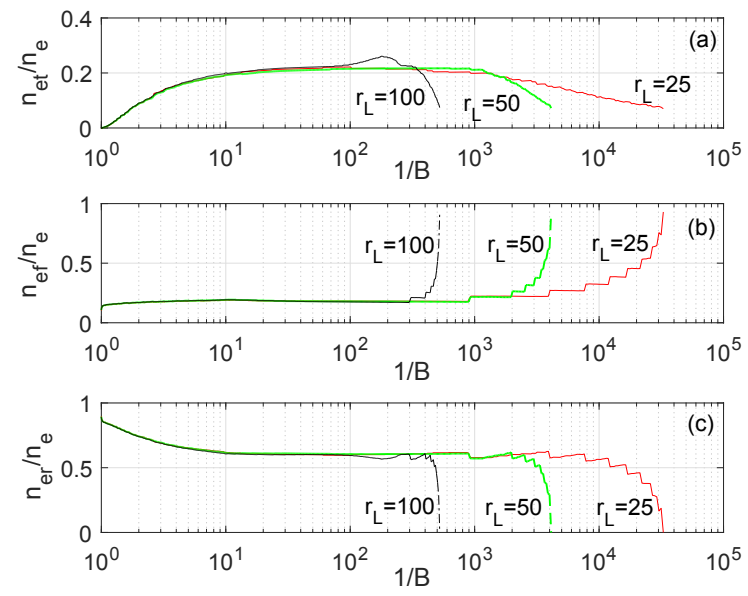

Figure 9. Panels (a), (b), and (c) show the electron densities of the trapped, free, and reflected electrons for $z_{M}=800$. Solid, dashed, and dot-dashed lines correspond to $r_{L}=100,50$, and 25 , respectively.

of the electrons in the steady state $F_{e}\left(z, v_{\|}, \mu, t_{F}\right)$ are known, the trajectories of the trapped particles can be computed as follows. First, by using the $z-\mu$ diagrams of $F_{e}\left(z, v_{\|}, \mu, t_{F}\right)$ for all the energies, we computed the distribution function $F_{e t}\left(z, v_{\|}, \mu\right)$ of the trapped electrons at the end of the simulation. Then, taking the values of $z$ and $v_{\|}$of the trapped electrons as initial conditions, we integrated numerically Eqs. 24-25 backward in time with the corresponding value of $\mu$ as a parameter and taking into account the computed time history of the accelerations. Panel (a) in Fig. 10 shows two examples of trapped orbits for $r_{L}=50$ and $z_{M}=800$. When analyzed from $t=t_{F}$ to $t=0$, they exhibit periodic motions that correspond to the trapped phases in the steady state, and then a segment that connects them to the entrance of the nozzle at the $z=z_{0}$. For one of the orbits, panel (a) shows the time at different points of the trajectory. We observe that this particular trapped electron is injected at $t=1287$ and the characteristic time to complete a periodic motion is $\sim 1500$. We also mention that, as $t \rightarrow \infty$, the orbits of the trapped particles are periodic because, once the stationary state has been reached and $\phi$ does not depend on time, Eqs. (24)-(25) are autonomous and integrable.

In our analysis, we computed the $10^{5}$ trapped trajectories with the highest values of the distribution function in the simulation for $r_{L}=50$ and $z_{M}=800$. From them, the values of the parallel and perpendicular velocities of the particles when they were injected at $z=z_{0}$ and the injection time $t_{i n j}$ were found. The color in panel (b) of Fig. 10 shows the value of the electron distribution function at $z=0$ given by Eq. (18) in the $v_{\| e}-v_{\perp e}$ plane, and the red dots the region of the phase that yields trapped trajectories in the interval $t_{i n j} \leq t<t_{F}$. The figure shows that there is a single phase space region for trapped particles in the injection and it is centered about $\left(v_{\| e}, v_{\perp e}\right) \approx(2,2)$. Almost the same region was found in the simulation with $r_{L}=25$ and $z_{M}=800$.

Each red point in panel (b) produces a set of trapped orbits during a certain 
interval of time. Beyond a certain time, particles cannot be trapped anymore because the system reaches the steady state and the orbits cannot connect with the trapped regions showed in Fig. 9. In order to investigate the injection time distribution of the trapped trajectories we constructed a histogram. As shown in panel (c) of Fig. 10, we summed the values of $\bar{f}_{e}$ of the particles trajectories that were injected within a certain temporal interval of width $\Delta t=200$. The histogram shows that particles injected at the very beginning of the simulation are not trapped, and the contribution of the trapped particles injected after $t \geq 4000$ is negligible. It reveals a complex behavior with several maxima and minima and highlights the non-linear character of the transient phase.
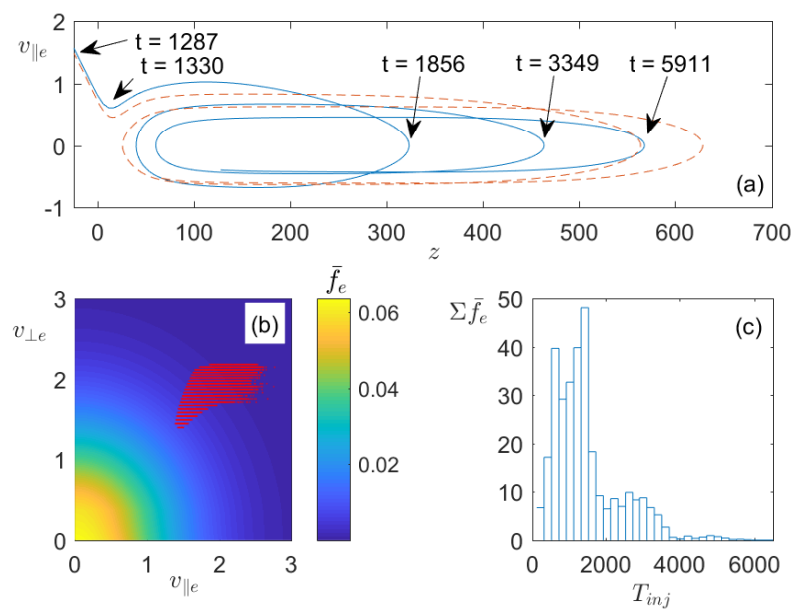

Figure 10. Trapped particles results for $r_{L}=50, z_{M}=800$, and $\phi_{M}=-2.75$. Panel (a), (b) and (c) shows two trapped trajectories, the region of the phase space at $z=0$ that yields trapped electrons within the time $T_{i n j} \leq t \leq T_{F}$, and the histogram of the injection time, respectively.

Since the trapping happens during the transient phase and there is an infinitude of possible scenarios describing the turn on of the nozzle, the natural question about the robustness of the previous results arises. This topic has been investigated by running a simulation with $r_{L}=25, z_{M}=800$, and the following boundary condition for the electrostatic potential

$$
\phi_{M}(t)=\phi_{0}+\left(\phi_{F}-\phi_{0}\right)\left\{\frac{1}{2}+\frac{1}{\pi} \arctan \left[\omega\left(t-t_{0}\right)\right]\right\},
$$

where we took the parameters $\phi_{0}=-5, \phi_{F}=-2.75, \omega=0.1$, and $t_{0}=2000$. Such a law keeps the potential at the exit of the simulation box roughly equal to $\phi_{0}=-5$ before $t_{0}=2000$, then makes a smooth transition to the current-free condition $\phi_{M}=-2.75$, and keeps this value constant afterwards. Interestingly, for this simulation, the maximum percentage of the density of trapped particles $(\sim 27 \%)$ is greater than the one obtained when $\phi_{M}$ is constant [about a $20 \%$ as shown by panel (a) in Fig. 11]. As shown in panels (b) and (c), the regions of the phase space at $z=z_{0}$ that are trapped at later times and the histogram are also different from the ones obtained for constant $\phi_{M}$. We then conclude that the steady state of a collisionless plasma expansion in a magnetic 
nozzle is not universal and depends on the particle history of the system. On the one hand, this justifies the uncertainties related to the trapped population in steady-sate models. On the other hand, this enhances the relevance of taking into account collisions, even if they are very rare, since they could be the mechanism leading to a final, unique distribution for the doubly-trapped population and a unique steady-state solution.
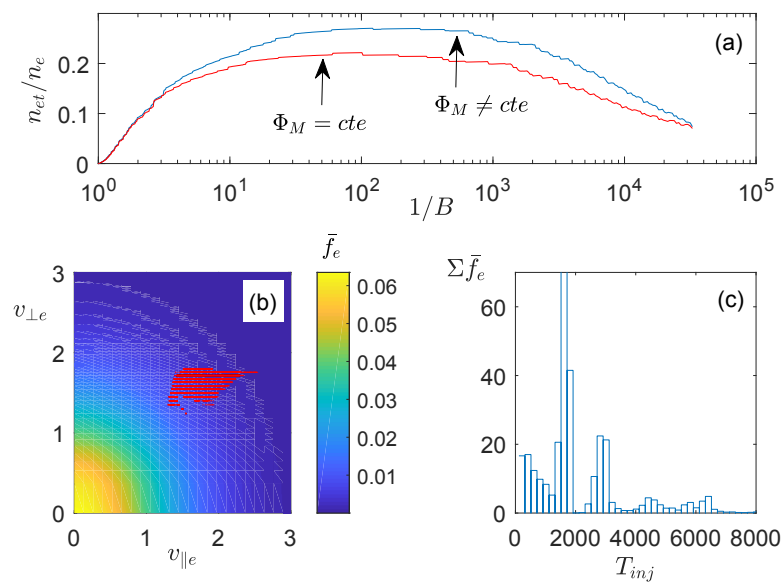

Figure 11. Trapped particles results for $r_{L}=25, z_{M}=800$, and $\phi_{M}$ given by Eq. 28. Panel (a), (b), and (c) show the relative density of the trapped population, the region of the phase space at $z=0$ that are trapped within the time $t_{i n j} \leq t \leq t_{F}$, and the histogram of the injection time, respectively. For comparison panel (a) also reproduces the relative density of the trapped population for the case $\phi_{M}=$ const.

\section{Conclusions}

The Vlasov-Poisson solver revealed interesting kinetic features of the unsteady paraxial expansion of collisionless plasma jets in magnetic nozzles. Its most relevant properties, i.e. its non-stationary character and the discretization of the Vlasov equation on a mesh in phase space, provided a self-consistent description of the particles trapped during the transient with a high accuracy. The parametric analysis showed how the electrostatic potential drop and the size of the simulation box should be selected to reproduce relevant physical conditions in the simulations. In general, the results are aligned with previous work based on stationary models, which considered a heuristic population of trapped particles. Such results include the electrostatic potential drop that yields a zero net current, the main features of the electron cooling (temperatures and heat fluxes), and the shape of the ion distribution function that is close to monoenergetic. Interestingly, the profiles within the quasineutral region of several quantities, such as the normal electron temperature and the particle densities, do not depend on the expansion rate if presented versus the inverse of the magnetic field.

The analysis provided quantitative information about the relative importance of the different electron populations. Reflected electrons are the dominant population in the 
quasineutral region, followed by the free and the trapped electrons. The latter represent about $20 \%$ of the total. This figure is much smaller than the one needed to make stationary Vlasov-Poisson solver converge in previous studies. Therefore, a population of trapped electrons seems to be a fundamental component of the expansion but it is not as large as considered earlier. This is one of the most important conclusions of this work. Moreover, it has been shown that the exact amount of trapped electrons depends on the particular history of the system and several steady states are possible.

The model of our work is restricted to the the paraxial and fully magnetized limit and therefore describes entirely the beam behavior at the axis of the nozzle only. These and other hypotheses are now revisited. Once the external magnetic field is fixed, the paraxial approximation adopted in this model, i.e. $R_{L}>>R_{0}$, imposes a constraint to the radius $R_{0}$ of the hole at the plasma-vacuum wall. Due to the divergence of the nozzle, it also limits the maximum axial distance that is meaningful in the numerical simulations. For a given magnetic field geometry, an estimation of such a distance can be found by computing the axial position where a magnetic line turns back. The paraxial hypothesis could be removed from the model by modifying the code slightly: Eq. 6 could be solved for many field lines and Eq. 8 should be extended to two-dimensional $(r$ and $z$ ) geometries. On the other hand, at some distance downstream, the local ion Larmor radius will become comparable to the jet radius and the ions will no longer be magnetized. Unlike its extension to two-dimensional geometries, the incorporation of de-magnetization effects to the code, which would involve transport of particles across different field lines, would not be straightforward.

As presently formulated, the code captures the adiabatic trapping of some electrons during the initial transient that leads to the development of a fully magnetized expansion to vacuum. Collisions are entirely neglected in this version, which is appropriate for the short time scale considered. However, this leaves open the question as to the ultimate steady state of the trapped electron population over the time scale of many collision times. The extension of the code to such scenario would require the computation at each time and location of the multiple integrals over velocities involved in the Boltzmann collision operator. Since for the usual weak collisionality case the new term will only be noticeable for times much longer than the initial transient time, it may be appropriate to neglect collisions until a reasonable steady state is reached and only then to turn collisions on and allow the gradual relaxation to the final steady state. This extension of the code would be an important contribution. It could clarify whether or not collisions erase completely the information about the transient, and if all the possible steady states of collisionless expansions collapse to a unique state.

\section{Acknowledgments}

G.S-A was supported by the Ministerio de Economía y Competitividad of Spain (Grant RYC-2014-15357). J.Z. was supported by Airbus DS (Grant CW240050). J.R. and M.M-S stays at UC3M for this research were supported by a UC3M-Santander Chair of 
Excellence and by National R\&D Plan (Grant ESP2016-75887), respectively. E.A. was supported by the MINOTOR project, that received funding from the European Unions Horizon 2020 research and innovation programme, under grant agreement 730028.

\section{Appendix A. Description of the numerical scheme}

The discretization in velocity space was carried out with $N_{v_{\|}} \times N_{\mu}$ points uniformly distributed as $\left(v_{\| j}^{\alpha}=-v_{\max }^{\alpha}+2(j-1) v_{\max }^{\alpha} /\left(N_{v_{\|}}-1\right)\right.$ and $\mu_{k}^{\alpha}=(k-1) \mu_{\max }^{\alpha} /\left(N_{m u}-1\right)$ with $j=1 \ldots N_{v_{\|}}$and $\left.k=1 \ldots N_{\mu}\right)$. The values of $v_{\max }^{\alpha}$ and $\mu_{\max }^{\alpha}$, which control the maximum ranges of the velocity mesh, depend on the specie and were chosen such that

$$
\frac{\beta_{\alpha}}{2}\left(v_{\| \max }^{\alpha}\right)^{2}=C_{v_{\|}} \delta_{\alpha}, \quad \mu_{\max }^{\alpha} \tilde{B}\left(z_{0}\right)=C_{\mu} \delta_{\alpha}
$$

with $C_{v_{\|}}$and $C_{\mu}$ two constants. In our simulations we set $C_{v_{\|}}=12.5$ and $C_{\mu}=8.95$, which was shown to be large enough (the value of the distribution functions were negligible close to the boundaries of the velocity mesh).

The distribution function $\bar{f}_{\alpha}\left(t_{m}, z_{i}, v_{\| j}^{\alpha} ; \mu_{k}^{\alpha}\right)$ at time $t_{m}$ was propagated to time $t_{m+1}=t_{m}+\Delta t$ with the splitting-time algorithm introduced Ref. [26]. It involves the following steps (the variable $\mu_{k}$ is omitted for brevity )

(i) Perform a half time step shift along the $z$-axis, $\bar{f}_{\alpha}^{*}\left(z, v_{\|}\right)=\bar{f}_{\alpha}\left(t_{m}, z-v_{\|} \Delta t / 2, v_{\|}\right)$.

(ii) Compute the acceleration $a_{\alpha}\left(z, t_{m}+\Delta t / 2\right)$ by using $\bar{f}_{\alpha}^{*}$ in 8 .

(iii) Perform a shift along the $v_{\|}$-axis, $\bar{f}_{\alpha}^{* *}\left(z, v_{\|}\right)=\bar{f}_{\alpha}^{*}\left[z, v_{\|}-a_{\alpha} \Delta \tilde{t}\right]$

(iv) Perform a half time step shift along the $z$-axis, $\bar{f}_{\alpha}\left(t+\Delta t, z, v_{\|}\right)=\bar{f}_{\alpha}^{* *}\left(z-\tilde{v}_{\|} \Delta t / 2, v_{\|}\right)$.

A key component of the code is the interpolation scheme, which is needed to make the shifts along $z$ and $v_{\|}$. After several tests monitoring the conservation of mass, momentum, and energy by using 11-13 and the results provided by the code, a cubic interpolation scheme for the $z$-shifts was selected. For electrons, $v_{\|}$-shifts were carried out with cubic splines, unless such operation is carried out at the three cells that adjoin the entrance and the exit of the simulation domain. For these cases, where the distribution function can be discontinuous due to the imposed boundary conditions, we used linear interpolation. The filamentation of the electron distribution function was avoided with the Fourier filter explained in [26]. For ions, spline interpolation in $v_{\|}$does not produce very accurate results due to the discontinuous character of the distribution function (note that no injected ions come back to the nozzle entrance). Linear interpolation for the $v_{\|}$interpolation of the ions gives more accurate results and was finally used in the simulations. The integrals in velocity space of the distribution functions, such us $n_{\alpha}$ in 9 , were carried out with a Sympson method. The two integrals in real space appearing in 8 were solved with a trapezoidal method.

The code was implemented with OpenMP (shared memory) in Fortran and the parallel computation took advantage of the conservation of $\mu$. The time propagation of a piece of the distribution function with a certain $\mu$-range was assigned to a specific processor. 


\section{Appendix B. Effect of the convergent segment}

Although this work is focused on the divergent segment of the nozzle, we here explain briefly why it is convenient to add a small convergent segment in the simulation domain, and the impact of its length, $\left|z_{0}\right|$, in the results of the expansion in the divergent region. A short parametric analysis for $z_{0}=-50,-25,-12.5$ and $r_{L}=50$, is presented next.

Panel (a) of Fig. B1 displays the normalized charge density profiles in stationary conditions. The sheath forms always at the left edge of the simulation box and its strength mitigates as the length of the convergent segment is increased [see inset in panel (a)]. Eventually the sheath would disappears if the whole plasma source were included, as it is the case in the stationary model of Ref. [19]. Panels (a) and (b) show that both the charge density and the electric potential (when referenced to the throat) are almost unaltered by the segment length (if this is larger than a few Debye lenghts). The same conclusion was reached for other plasma properties in the divergent nozzle, like for instance the temperature profiles. In this respect, the results of our work are robust. If the left edge of the simulation were placed at the throat, a nonnegligible sheath would appear next to the throat affecting significantly the potential fall along the divergent nozzle. It is clear that there are distribution functions that avoid the development of a sheath at the left edge, but we do not see a way to characterize them. The presence of this spurious sheath has been reported also in full particle-in-cell simulations of unmagnetized and magnetized plumes too $[27,28]$
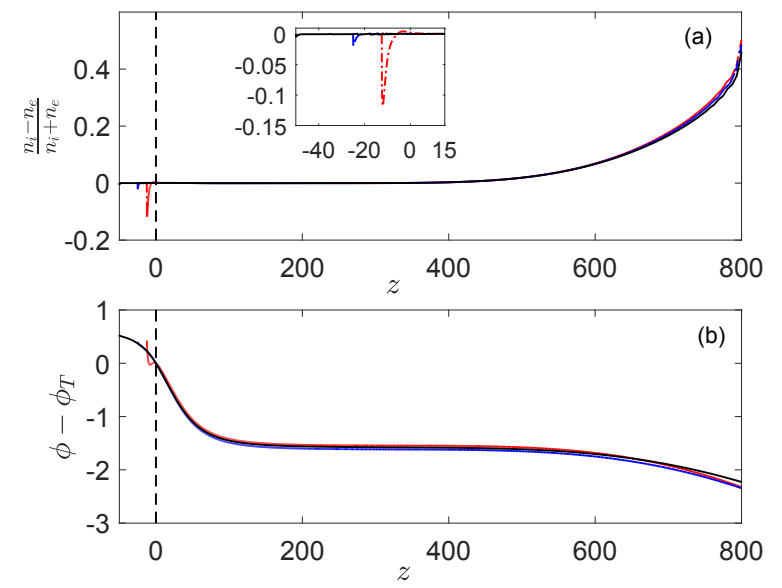

Figure B1. Panels (a) and (b) show the charge densities and potential profiles for simulations with lengths of the convergent segment equal to $z_{0}=-50$ (solid black), $z_{0}=-25$ (dashed blue) and $z_{0}=-12.5$ (red dashed-dotted). The curves practically overlap. 
[1] Alice K. Harding. The neutron star zoo. Frontiers of Physics, 8(6):679-692, 2013.

[2] Brian E. Wood. Astrospheres and solar-like stellar winds. Living Reviews in Solar Physics, 1(1):2, 2004.

[3] Stephen P. Reynolds. Supernova remnants at high energy. Annual Review of Astronomy and Astrophysics, 46:89-126, 2008.

[4] X. L. Zhang, R. S. Fletcher, S. L. Rolston, P. N. Guzdar, and M. Swisdak. Ultracold plasma expansion in a magnetic field. Phys. Rev. Lett., 100:235002, Jun 2008.

[5] W.-M. Wang, P. Gibbon, Z.-M. Sheng, and Y.-T. Li. Magnetically assisted fast ignition. Physical Review Letters, 114(1), 12015.

[6] S. A. Andersen, V. O. Jensen, P. Nielsen, and N. D'Angelo. Continuous supersonic plasma wind tunnel. The Physics of Fluids, 12(3):557-560, 1969.

[7] C. Charles, R. W. Boswell, and M. A. Lieberman. Xenon ion beam characterization in a helicon double layer thruster. Applied Physics Letters, 89(26):261503, 2006.

[8] Mariano Andrenucci. Magnetoplasmadynamic Thrusters. John Wiley \& Sons, Ltd, 2010.

[9] Alexey V. Arefiev and Boris N. Breizman. Theoretical components of the vasimr plasma propulsion concept. Physics of Plasmas, 11(5):2942-2949, 2004.

[10] S. A. Cohen, N. S. Siefert, S. Stange, R. F. Boivin, E. E. Scime, and F. M. Levinton. Ion acceleration in plasmas emerging from a helicon-heated magnetic-mirror device. Physics of Plasmas, 10(6):2593-2598, 2003.

[11] Kazunori Takahashi, Aiki Chiba, Atsushi Komuro, and Akira Ando. Experimental identification of an azimuthal current in a magnetic nozzle of a radiofrequency plasma thruster. Plasma Sources Science and Technology, 25(5):055011, 2016.

[12] Y Takama and K Suzuki. Experimental studies on nonequilibrium plasma flow in a convergentdivergent magnetic field. Plasma Sources Science and Technology, 17(1):015005, 2008.

[13] Donal L. Chubb. Fully ionized quasi-one-dimensional magnetic nozzle flow. AIAA Journal, 10(2):113-114, 1972.

[14] Alexey V. Arefiev and Boris N. Breizman. Magnetohydrodynamic scenario of plasma detachment in a magnetic nozzle. Physics of Plasmas, 12(4):043504, 2005.

[15] E. Ahedo and M. Merino. Two-dimensional supersonic plasma acceleration in a magnetic nozzle. Physics of Plasmas, 17(7):073501, 2010.

[16] Mario Merino and Eduardo Ahedo. Plasma detachment in a propulsive magnetic nozzle via ion demagnetization. Plasma Sources Science and Technology, 23(3):032001, 2014.

[17] Mario Merino and Eduardo Ahedo. Effect of the plasma-induced magnetic field on a magnetic nozzle. Plasma Sources Science and Technology, 25(4):045012, 2016.

[18] Heath Lorzel and Pavlos G. Mikellides. Three-dimensional modeling of magnetic nozzle processes. AIA A Journal, 48(7):1494-1503, 2010.

[19] M. Martinez-Sanchez, J. Navarro-Cavallé, and E. Ahedo. Electron cooling and finite potential drop in a magnetized plasma expansion. Physics of Plasmas, 22(5):053501, 2015.

[20] Juan Ramón Sanmartín Losada. Active charging control and tethers. In C.P. Catani, editor, Space environment : prevention of risk related to spacecraft charging : Space technology course, pages 515-533. Cépadues, Tolouse, 2002.

[21] G. Sánchez-Arriaga and D. Pastor-Moreno. Direct vlasov simulations of electron-attracting cylindrical langmuir probes in flowing plasmas. Physics of Plasmas, 21(7):073504, 2014.

[22] A. V. Gurevich. Distribution of Captured Particles in a Potential Well in the Absence of Collisions. Soviet Journal of Experimental and Theoretical Physics, 26:575, March 1968.

[23] M A Raadu. Expansion of a plasma injected from an electrodeless gun along a magnetic field. Plasma Physics, 21(4):331, 1979.

[24] Albert Meige, Rod W. Boswell, Christine Charles, and Miles M. Turner. One-dimensional particlein-cell simulation of a current-free double layer in an expanding plasma. Physics of Plasmas, 12(5):052317, 2005.

[25] M. Martinez-Sanchez and E. Ahedo. Magnetic mirror effects on a collisionless plasma in a 
convergent geometry. Physics of Plasmas, 18(3):033509, 2011.

[26] C.Z Cheng and Georg Knorr. The integration of the vlasov equation in configuration space. Journal of Computational Physics, 22(3):330 - 351, 1976.

[27] Yuan Hu and Joseph Wang. Fully kinetic simulations of collisionless, mesothermal plasma emission: Macroscopic plume structure and microscopic electron characteristics. Physics of Plasmas, 24(3):033510, 2017.

[28] Min Li, Mario Merino, Eduardo Ahedo, Junxue Ren, and Haibin Tang. Full-pic code validation and comparison against fluidmodels on plasma plume expansions. In Proceedings of International Electric Propulsion Conference, pages IEPC-2017-230. Georgia Institute of Technology, Oct 2017. 